\title{
Neutral Earthing Reactance Design in Mitigating Third Harmonic Current at the Generator Neutral
}

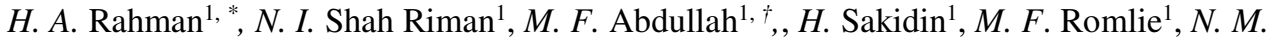 \\ Nor $^{1}$
}

${ }^{1}$ Electrical and Electronic Engineering Department, Universiti Teknologi PETRONAS, 32610, Bandar Seri Iskandar, Perak, Malaysia.

\begin{abstract}
Third harmonic $(\mathrm{TH})$ current from salient-pole synchronous generator that return to its neutral via cable capacitance or other neutral grounding points in the network can cause the neutral earthing resistor (NER) to experience high temperature. Generator neutral earthing reactance (NERX) can be used to mitigate the TH current flowing in the neutral under normal condition. This paper aims to study the suitable design of NERX to be implemented in Universiti Teknologi PETRONAS (UTP) distribution system to reduce the TH current at the generator neutral. The model of UTP distribution system is developed using MATLAB Simulink in order to simulate the flow of TH current. NERX is then designed and implemented to the system to mitigate the magnitude of TH current. Various values of NERX are applied to the system as the neutral current and fault current are recorded and graphed. The application of $0.175 \mathrm{H}$ NERX increased the TH neutral current in island mode and normal operation to $33.18 \%$. However, the NERX causes the TH fault current to reduce to $44.33 \%$ under fault. For parallel mode, $78.27 \%$ of TH neutral current and also $42.91 \%$ of TH fault current are reduced under normal operation and fault respectively.
\end{abstract}

\section{Introduction}

Power quality is defined as part of the field in power engineering that involves modelling, analysis and mitigation of any difficulties related to current and voltage distortion in power system to increase the system reliability [1]. In power quality problem, power harmonic is one of primary concern as the type of current and voltage waveform distortion mainly caused by switching device such as rectifier and power electronic switches [2].

The frequency of harmonic element is the multiplication of fundamental frequency and it can exist up to more than 50th harmonic. However, due to the higher inductive reactance that proportional to frequency in power system network, the harmonics above 50th are negligible [3].

Studies are focusing on the odd order of harmonics since the even order of harmonics mostly cancels each other in three phase system due to symmetrical waveform. Referring to the odd-numbered harmonics, an analysis done in [4] suggests that these parts of harmonics

\footnotetext{
* Corresponding author: hadamar150793@gmail.com

$\uparrow$ Corresponding author: mfaris_abdullah@utp.edu.my
} 
can exist in positive, negative and zero sequences in accordance to their fundamental frequency theory. Zero sequence harmonic or also known as triplen harmonic, consists of 3rd, 9th, 15th, 21st etc. are considered as the most problematic in three-phase system [3]. At the generation side, salient-pole synchronous generator is one of the triplen harmonic sources. In salient-pole synchronous generator construction, the pitch factor, distribution and slot skew are associated with triplen harmonic voltage formation. Under no load condition, third harmonic $(\mathrm{TH})$ voltage is produced due to the salient-pole shape and the concentrated field winding. In balanced load, the field current, salient-pole shape, direct-axis and quadratureaxis armature reaction are the components that contribute to the generation of TH component [5]. TH current can only exist within the system in the availability of $\mathrm{TH}$ voltage and zero sequence network [6].

A study in [6] stated that triplen harmonic current had caused the Neutral Earthing Resistor (NER) of synchronous generator to experience high temperature. Due to zero sequence in nature, the magnitude of triplen harmonics current sum up to three times the phase triplen harmonics current magnitude at the neutral of synchronous generator [7]. Moreover, in a three-phase system with $60 \mathrm{~Hz}$ fundamental frequency, the TH with harmonic frequency of $180 \mathrm{~Hz}$ adds cumulatively in the neutral conductor up to $173 \%$ of phase current [3].

$\mathrm{TH}$ voltage that exists in the generator had caused the TH current to circulate through the generator stator, the neutral grounding device on the generator, and other grounded system device [8]. Ground current sources such as wye-delta transformer or grounded generator that are connected with the same voltage level to another neutral grounded generator will cause the TH current to circulate between them [9].

Synchronous generator TH propagation model is developed in [10]. According to [8], the zero sequence reactance of synchronous generator for the TH is assumed to be three times of the fundamental frequency zero sequence reactance due to their frequency dependence and the neutral impedances are multiplied by three to convert them to zero sequence network. From the analysis done in [10] for synchronous generator that is connected in parallel with the utility grid under balanced load, the TH circulates between the generator and the transformer at utility substation through both NER path.

In some cases, the TH current from the generator returns to its neutral through cable capacitance or other neutral grounding points in the network especially for the transmission system that utilise underground cables. Underground cables have high line capacitance between the core and earth point with mutual inductance [11]. Due to the nature of underground cables, TH current can return to the generator neutral via the capacitance of underground cables that provides the earth path [6], [12].

Neutral Earthing Impedance (NEI) typically used as grounding device at generator or transformer neutral to reduce earth fault current to a safer level. NEI can either be in the form of a resistor or a reactor. NEI with high resistance grounding which is also known as NER has the advantage on limiting the steady state and severe transient overvoltage aside from allowing a greater reduction in fault current value. NEI that is purely inductive reactance has limitation in reducing the earth fault current due to transient overvoltage concerns. This type of NEI is also called Neutral Earthing Reactance (NERX) and usually used in a system that allows the earth fault current to be at least $25 \%$ of the three-phase fault current [13].

Simulation conducted in [14], it is stated that adding inductor to the grounding system can minimised the neutral current. Hence, the objective of this paper is to simulate the effect of NERX by using various value of inductor as reactor grounding toward mitigation of TH current at the generator neutral as indicated by the problem statement in section II. Therefore, Universiti Teknologi PETRONAS (UTP) distribution system model was developed using MATLAB Simulink in order to simulate the flow of TH current and NERX with various inductor values were implemented to the model developed as described in the methodology 
in section III. The simulation results are presented and discussed in section IV. Finally, the conclusion and recommendation are summarised in section $\mathrm{V}$.

\section{Problem Statement}

TH current continuously flow from synchronous generator and return to its neutral during steady state condition via neutral or ground path has caused the NER to experience high temperature at UTP Gas District Cooling (GDC) plant. Investigation done in [6] associated to the problem stated that the highest generator NER temperature rise occur during one generator in parallel with utility grid, Tenaga Nasional Berhad (TNB) because of significant increase in neutral current associated with triplen harmonic currents.

The problem exists primarily due to TH current as higher harmonics order of the triplen harmonics have smaller harmonic voltage been produced by generator. In parallel mode, the zigzag transformer at TNB substations provide the lowest zero sequence impedance for TH current returning to the generator neutral [6]. The long-term effect of generator NER overheating is the degradation of the insulation lifespan which can lead to failure. Therefore, a proper grounding method at the generator needs to be implemented in order to minimise the TH current as being the highest among the triplen harmonic currents in both island mode and parallel mode with TNB.

\section{Methodology}

In designing the NERX that can be used to mitigate the high TH current at the generator neutral, it should be noted that the existing design of NER rating needs to be taken into consideration for suitable implementation in the system. In other word, the NERX designed must not compromise the existing system design for earth fault current mitigation.

\subsection{UTP Distribution System Model}

Modelling of UTP distribution system is done in MATLAB Simulink software utilising the available Simpower/Simscape blocks. The UTP distribution system is modelled in two mode of operations; island mode and parallel mode with TNB grid. In the existing system, NER at the generator is used to limit the earth fault current to $200 \mathrm{~A}$. In modelling the generator, Three-Phase Voltage Source block is used to represent the gas turbine generator (GTG-A and GTG-B) and the TNB source. Both GTG-A and GTG-B rated $11 \mathrm{kV}, 6.625 \mathrm{MVA}$ and the value of the NER used is $31.75 \Omega$. The wye winding of $30 \mathrm{MVA}, 132 / 11 \mathrm{kV}$, step down transformer at TNB substation is earthed through $4 \Omega$ NER. TNB source at $132 \mathrm{kV}$ is connected to delta winding of this transformer. Three-Phase Transformer (two windings) block is used to model the transformer with appropriate parameters.

To model the cable as a short length category, a 'Pi Section Line' Simulink block is used to represent one conductor. The Pi section is typically used for medium length transmission. However, due to the nature of underground cable construction with high line capacitance between the core and earth point, the Pi Section Line block need to be used in this scenario. UTP distribution system consists of XLPE insulated underground power cable. To model a three-core underground cable, three 'Pi Section Line' blocks are needed for each phases. In modelling the UTP medium voltage cable for short length category, only one Pi section is used for each block and the cable parameters are stipulated in Table 1. In UTP distribution system, various cable sizes are used from one bus to another. Therefore, the cable sizes tabulated in Table 1 are used in the model according to the single line diagram of UTP 
distribution system. The simplified model of the system in island and parallel mode of operation are shown in Fig. 1 and Fig. 2 respectively.

According to [15], TH source can be modelled as independent AC voltage source for each phase. In three phase system, three independent $\mathrm{AC}$ voltage sources at the $\mathrm{TH}$ frequency are connected in series with the generators' phases to represent the TH voltage sources. Fig. 3 shows the series connection of the AC voltage source having the TH frequency with ThreePhase Voltage Source block as the fundamental source. Table 2 shows the TH harmonic voltage used in each phase for both modes at GTG-A which is tuned to produce an approximate third harmonic current with the measured value as in Table 3 from the analysis done in [6]. This TH model will be applied to the UTP distribution system model.

Table 1. XLPE insulated power cable parameter (copper conductor).

\begin{tabular}{|c|c|c|c|c|}
\hline \multirow{2}{*}{$\begin{array}{c}\text { Type of } \\
\text { Cable }\end{array}$} & Cable size & $\begin{array}{c}\text { Resistance per } \\
\text { kilometer } \\
(\mathrm{ohm} / \mathrm{km})\end{array}$ & $\begin{array}{c}\text { Inductance per } \\
\text { kilometer } \\
(\mathrm{mH} / \mathrm{km})\end{array}$ & $\begin{array}{c}\text { Capacitance per } \\
\text { kilometer } \\
(\mathrm{uF} / \mathrm{km})\end{array}$ \\
\hline \multirow{3}{*}{$\begin{array}{c}\text { Three- } \\
\text { Core }\end{array}$} & 70 & 0.3244 & 0.3374 & 0.3 \\
\cline { 2 - 5 } & 240 & 0.128 & 0.29285 & 0.43 \\
\cline { 2 - 5 } & 300 & 0.0984 & 0.2833 & 0.48 \\
\hline \multirow{2}{*}{$\begin{array}{c}\text { Single- } \\
\text { Core }\end{array}$} & 400 & 0.0797 & 0.27375 & 0.53 \\
\hline
\end{tabular}

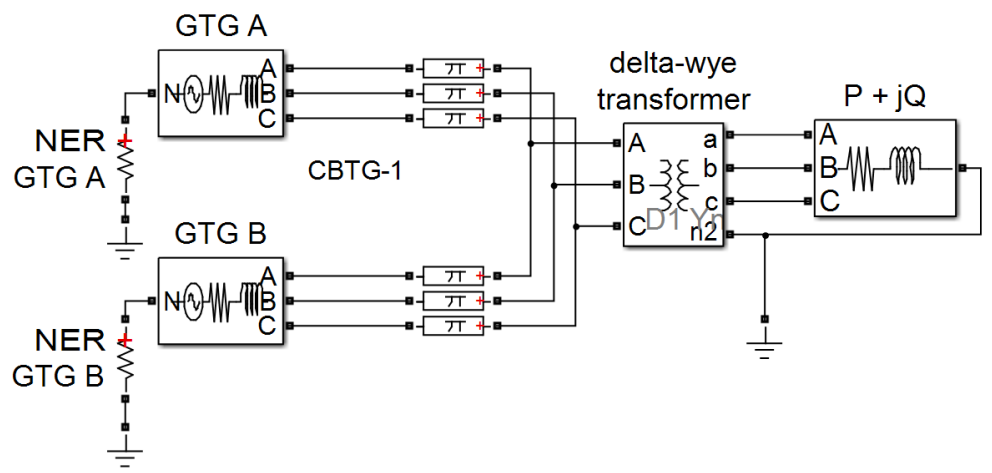

Fig. 1. Simplified UTP distribution network in island mode.

Table 2. TH voltage used for TH source.

\begin{tabular}{|l|l|l|c|}
\hline \multirow{4}{*}{ GTG-A } & \multirow{3}{*}{ Island mode } & TH Voltage Phase A (V) & 400 \\
\cline { 3 - 4 } & & TH Voltage Phase B (V) & 400 \\
\cline { 3 - 4 } & \multirow{3}{*}{$\begin{array}{l}\text { Parallel } \\
\text { mode }\end{array}$} & TH Voltage Phase C (V) & 400 \\
\cline { 3 - 4 } & & TH Voltage Phase A (V) & 260 \\
\cline { 3 - 4 } & TH Voltage Phase B (V) & 260 \\
\hline
\end{tabular}




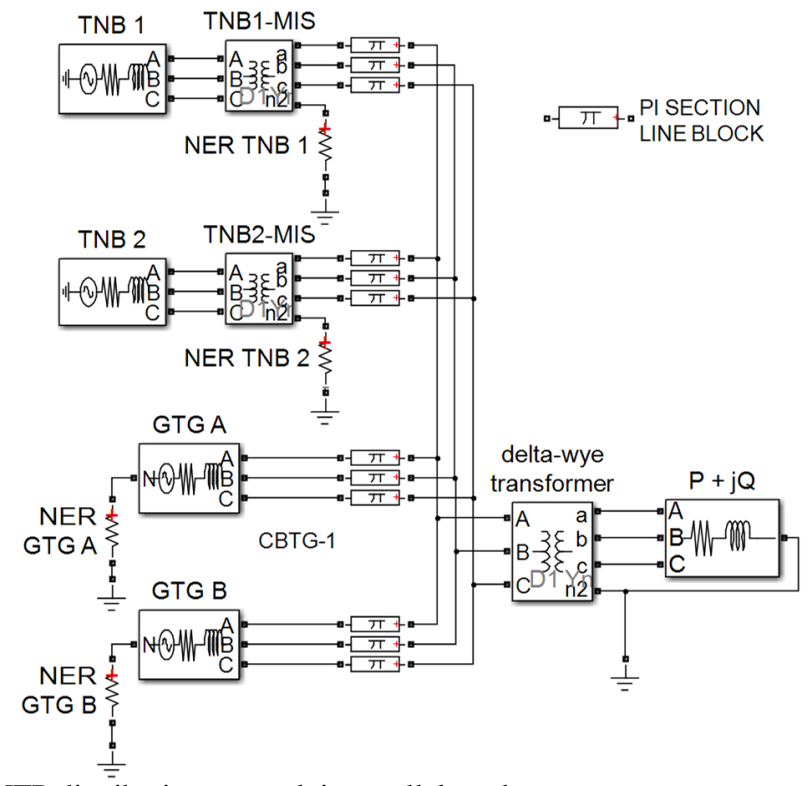

Fig. 2. Simplified UTP distribution network in parallel mode.

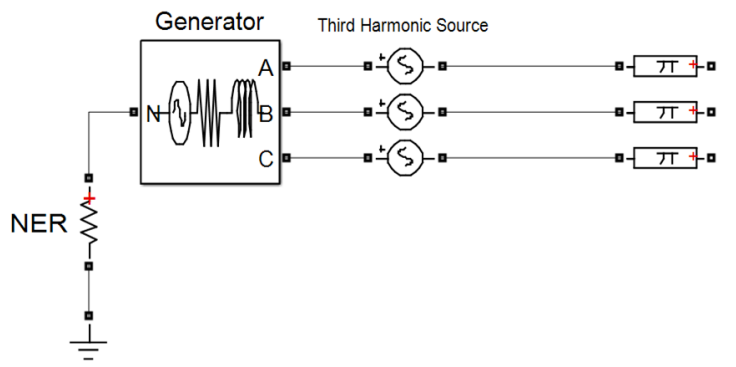

Fig. 3. TH source implementation.

Table 3. Simulated TH neutral current.

\begin{tabular}{|l|c|c|}
\hline $\begin{array}{l}\text { GTG-A } \\
\text { Neutral line }\end{array}$ & $\begin{array}{c}\text { Simulated TH neutral } \\
\text { current (A) }\end{array}$ & $\begin{array}{c}\text { Measured TH } \\
\text { neutral current } \\
\text { (A) }\end{array}$ \\
\hline Island mode & 3.587 & 3.6 \\
\hline Parallel mode & 7.114 & 7.1 \\
\hline
\end{tabular}

\subsection{UTP Distribution System Simulation}

Simulation started with the existing system in island mode and normal condition utilising NER to obtain the neutral current value. Fourier function blocks in the MATLAB Simulink model are set to TH frequency and used to measure the peak magnitude of the TH current and voltage at the generator neutral. Once obtained, the system is simulated with single-phase to ground fault at CBTG-1 bus as shown in Fig. 4 since single-phase to ground fault will yield the highest ground current. The fault current measured will be used as constant variable to determine the suitable inductor value for NERX. The similar steps are used to obtain the neutral current and fault current for the system in parallel with TNB grid.

The NERs are then replaced with NERXs in the system simulation as shown in Fig. 5 and Fig. 6 for island and parallel mode of operation respectively. The inductor value is varied 
from $0 \mathrm{H}$ to $1 \mathrm{H}$ and the neutral current passing through NERX is observed and recorded for both island and parallel mode of operation under normal condition. Single-phase to ground fault is then simulated at CBTG-1 bus for each inductor value and the fault currents are recorded.

The site data measurement from UTP system is used in the voltage source model magnitude for fundamental frequency and TH. In verifying the model, results of simulation are compared with the measured neutral current of existing system using NER under normal condition.

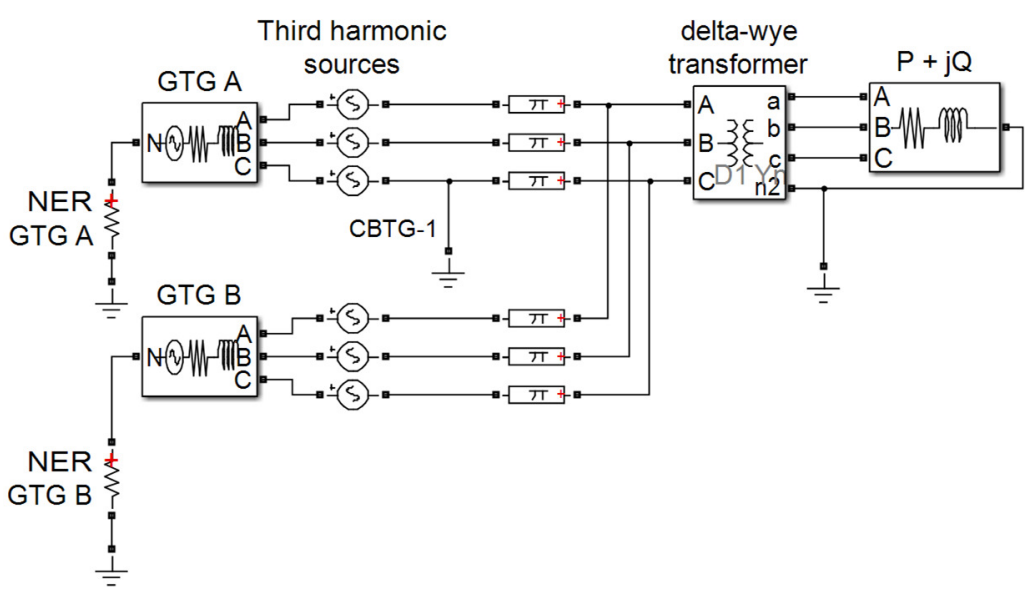

Fig. 4. Single-phase to ground fault at CBTG-1.

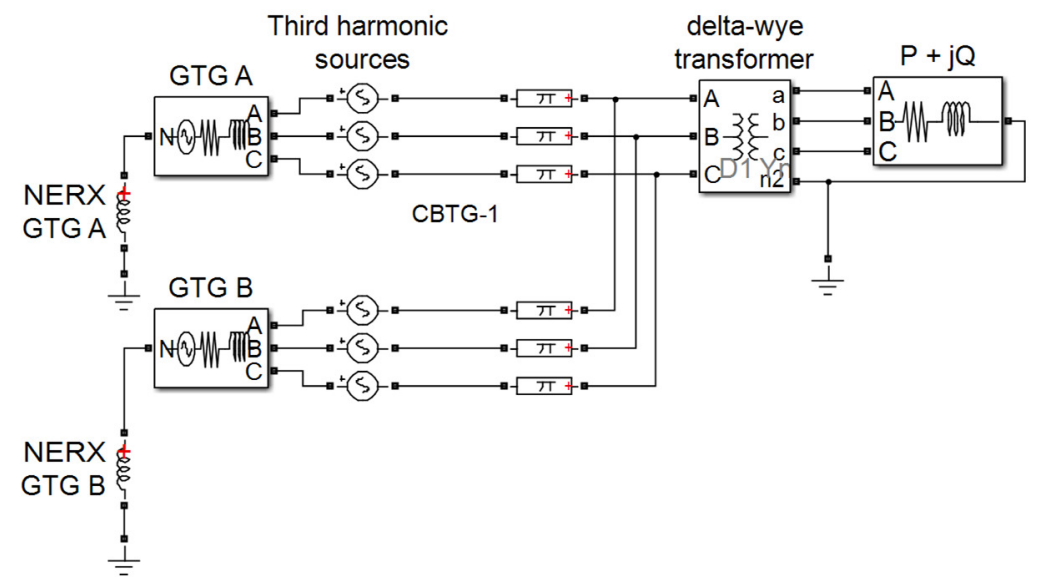

Fig. 5. Implementation of NERX at generators neutral in island mode. 


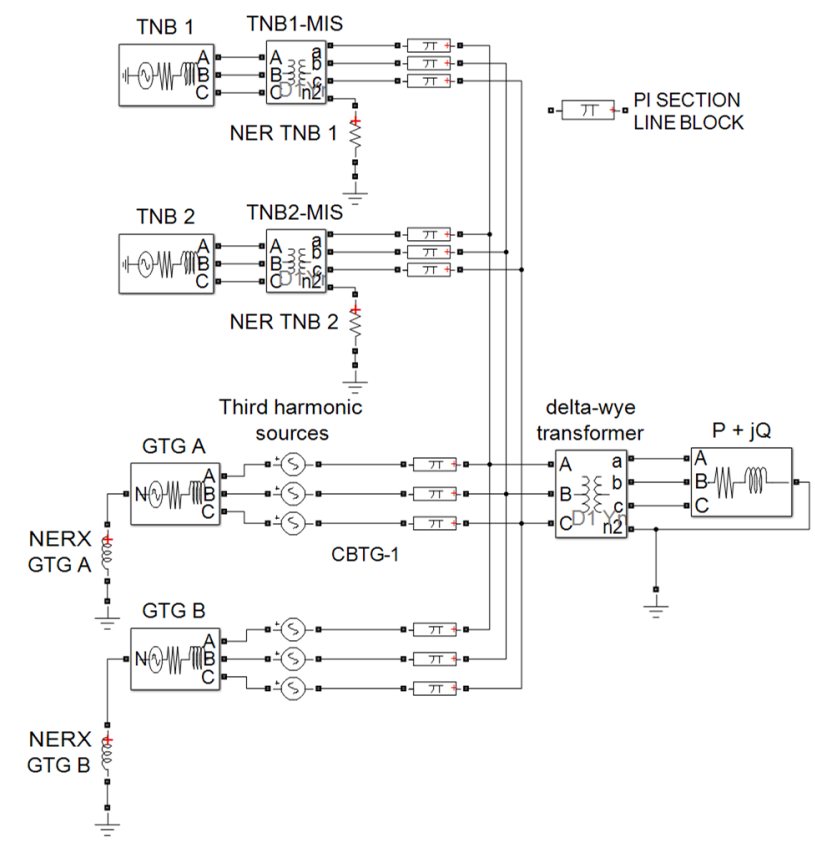

Fig. 6. Implementation of NERX at generators neutral in parallel mode.

\section{Result and Discussion}

The results from simulations are divided into island and parallel mode of operation. It is important to note that the equivalent reactor value at fundamental frequency for $31.75 \Omega$ NER is $0.1 \mathrm{H}$. As the reactor grounding is implemented into the simulation and its value is varied, all recorded readings are graphed to show the characteristic of the neutral current for each mode of operations. Subsequently, the fault current when single-phase to ground fault occur at CBTG-1 bus is simulated and observed. Under normal condition there should not be any fundamental neutral current since load are always balanced at medium voltage system. Hence, neutral current at normal condition is referring to $\mathrm{TH}$ current alone by default. However, during single-phase to ground fault, the fault or neutral current contained both fundamental frequency and TH currents. Since both GTG-A and GTG-B are identical, only simulation results of GTG-A are presented here.

\subsection{Island mode}

Table 4 shows the simulation result of neutral current in normal and during single-phase to ground fault event at CBTG-1 bus for the system utilising the existing NER in island mode. These values are used as constant variable in comparative analysis between NER and NERX. Fig. 7 shows the plot of neutral current for GTG-A versus reactor value in island mode. In this plot, a significant increase in neutral current is observed as reactor value reaches $0.09 \mathrm{H}$ and decreases drastically after this value. One of the possible reason for this to happen is the occurrence of series resonance between NERX and the system shunt capacitance causing the zero sequence impedance value to drop to minimum. The plotted result of simulated fault current at CBTG-1 of GTG-A versus reactor value is shown Fig. 8. As the NERX value varies from $0 \mathrm{H}$ to $1 \mathrm{H}$, an exponential decrease characteristic is observed from the plot. 
Table 4. Simulated TH neutral current.

\begin{tabular}{|c|c|c|c|}
\hline \multirow{2}{*}{$\begin{array}{c}\text { NER } \\
(\Omega)\end{array}$} & \multirow{2}{*}{ Generator } & $\begin{array}{c}\text { Neutral Current (A) } \\
\text { gingle-phase to } \\
\text { ground fault }\end{array}$ & Normal \\
\hline 31.75 & GTG-A & 190.6 & 3.587 \\
\hline
\end{tabular}

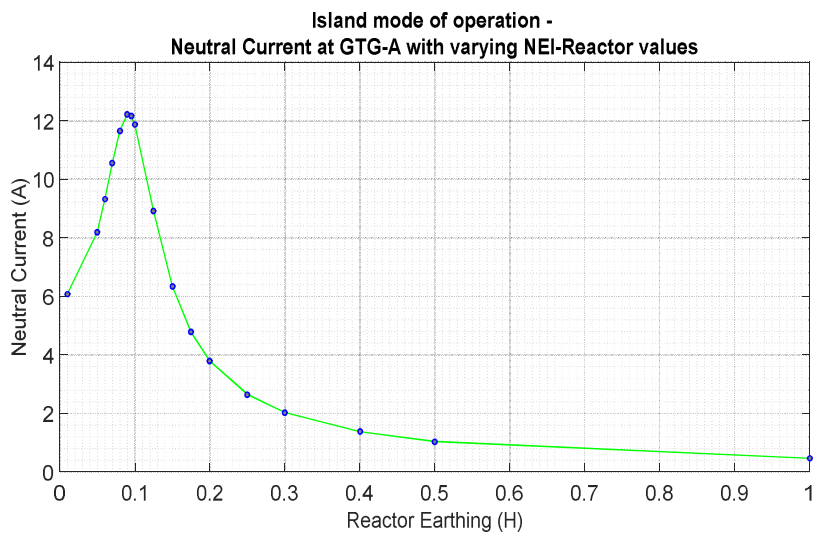

Fig. 7. Neutral current at GTG-A vs reactor value (Island mode).

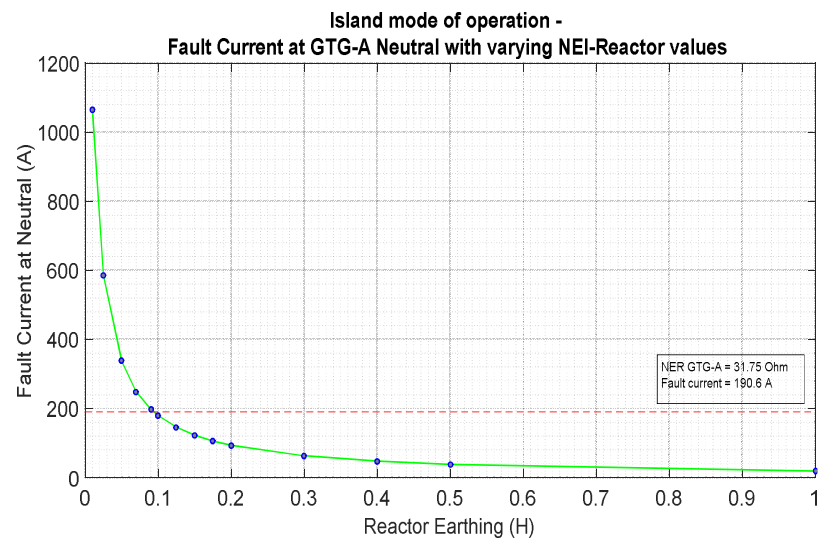

Fig. 8. Fault current at GTG-A neutral vs reactor value (Island mode).

\subsection{Parallel mode}

In parallel mode of operation, the simulation result of the system using the existing NER is shown in Table 5. Fig. 9 shows the plot of neutral current for GTG-A versus reactor value of the system in parallel operation under normal condition. In this plot, the neutral current at GTG-A decreases exponentially as reactor value is varied from $0 \mathrm{H}$ to $1 \mathrm{H}$. There is no sign of series or parallel resonance in parallel mode. The simulated single-phase to ground fault current versus reactor value in parallel operation is also plotted in Fig. 10 and the plot shows a similar characteristic as in Fig. 8 during island mode. 
Table 5. Simulated TH neutral current.

\begin{tabular}{|c|c|c|c|}
\hline \multirow{2}{*}{$\begin{array}{c}\text { NER } \\
(\Omega)\end{array}$} & \multirow{2}{*}{ Generator } & \begin{tabular}{c} 
Neutral Current (A) \\
\cline { 3 - 4 } \\
gingle-phase to \\
ground fault
\end{tabular} & Normal \\
\hline 31.75 & GTG-A & 175.7 & 7.114 \\
\hline
\end{tabular}

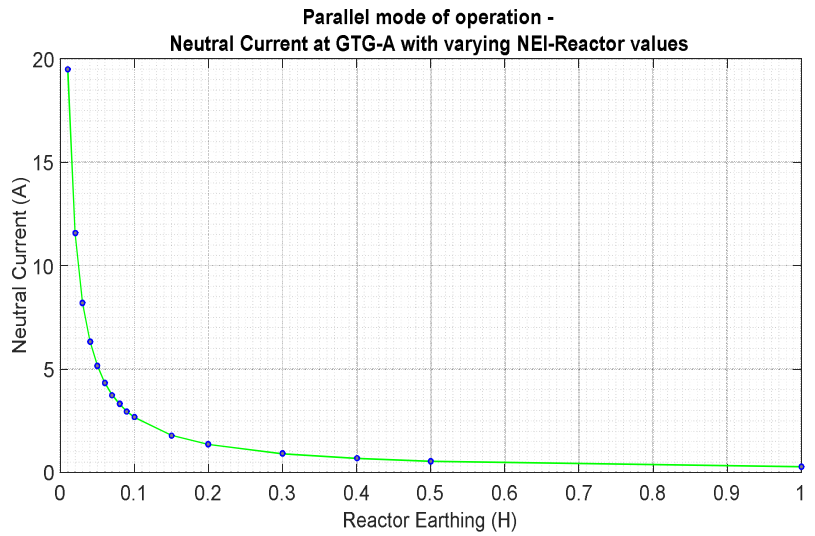

Fig. 9. Neutral current at GTG-A vs reactor value (Parallel mode).

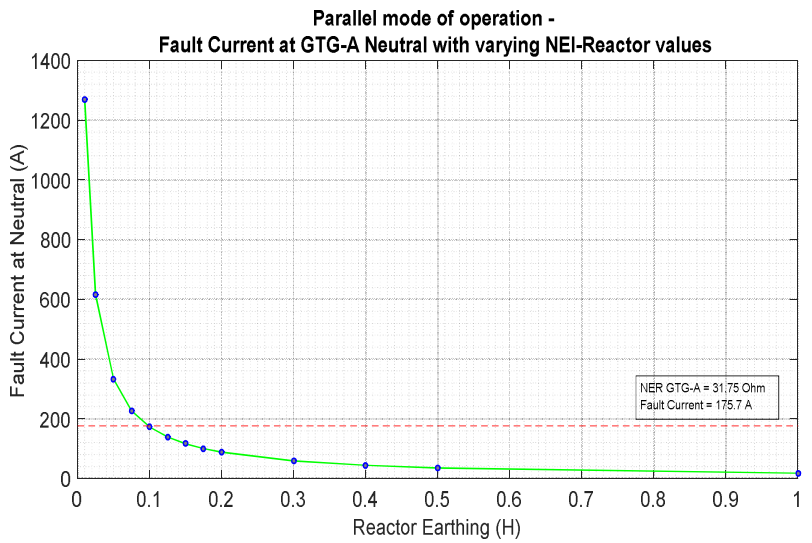

Fig. 10. Fault current at GTG-A neutral vs reactor value (Parallel mode).

The plotted graph in Fig. 7, 8, 9 and 10 are used in determining the suitable NERX value to be implemented into the system. The following conditions must be met in choosing the reactor value:

1) The fault current at generator neutral using NERX must be limited not more than the generator full load current of 348 A for both mode of operation. However, too low single-phase to ground will also cause the protection relay unable to pick-up and operate during high resistance fault.

2) The normal neutral current at generator must be mitigated down to a suitable value in both mode of operation.

Referring to the conditions and the plotted graphs from Fig. 7 to 10, the reactor value of $0.15 \mathrm{H}$ is chosen as suitable NERX for both GTG-A and GTG-B. The impedance value of 
the chosen NERX is $54.98 \Omega$ at fundamental frequency. In mitigating TH current, the chosen reactor will be having an impedance value of $164.93 \Omega$ due to TH frequency.

Comparative analysis for island and parallel mode of operation is tabulated in Table 6 , Table 7, Table 8 and Table 9 for the chosen NERX value. In island mode, the reactor value of $0.175 \mathrm{H}$ has caused $33.18 \%$ increase of the TH current at GTG-A neutral. In parallel mode, it is observed that using the chosen value of NERX, TH current reduce significantly by $78.27 \%$ at GTG-A neutral.

In term of earth fault current limitation at the generator neutral, using the value of 0.175 $\mathrm{H}$ as NERX has caused the fault current to decrease in both mode of operation as shown in Table 8 and Table 9. Nonetheless, the reduction in earth fault current is still within the tolerable range for the system. TH current at neutral can be further reduced by using larger reactor value. However, the fault current will also decreases and a smaller fault current at neutral can cause sensitivity issue of the protection relay operation to detect earth fault.

Table 6. Percentage reduction/increase of neutral current between NER and selected NERX - island mode.

\begin{tabular}{|c|c|c|c|}
\hline \multirow{2}{*}{$\begin{array}{l}\text { Generators in } \\
\text { Island mode }\end{array}$} & \begin{tabular}{c}
$|c|$ \\
NER \\
\cline { 2 - 3 }
\end{tabular} & $\begin{array}{c}\text { TH Neutral Current (A) } \\
\text { NERX }\end{array}$ & $\begin{array}{c}\text { Reduction/ } \\
\text { Increase (\%) }\end{array}$ \\
\hline GTG-A & $31.75 \Omega)$ & $(0.175 \mathrm{H}$ or TH = 164.93 $\Omega)$ & $+33.18 \%$ \\
\hline
\end{tabular}

Table 7. Percentage reduction/increase of fault current between NER and selected NERX - island mode.

\begin{tabular}{|c|c|c|c|}
\hline \multirow[b]{2}{*}{$\begin{array}{l}\text { Generators in } \\
\text { Island mode }\end{array}$} & \multicolumn{2}{|c|}{ Fault Current (A) } & \multirow[b]{2}{*}{$\begin{array}{l}\text { Reduction/ } \\
\text { Increase (\%) }\end{array}$} \\
\hline & $\begin{array}{c}\text { NER } \\
(31.75 \Omega)\end{array}$ & $\begin{array}{c}\text { NERX } \\
(0.175 \mathrm{H} \text { or at fundamental }= \\
54.98 \Omega)\end{array}$ & \\
\hline GTG-A & 190.6 & 106.1 & $-44.33 \%$ \\
\hline
\end{tabular}

Table 8. Percentage reduction/increase of neutral current between NER and selected NERX - parallel mode.

\begin{tabular}{|c|c|c|c|}
\hline \multirow{2}{*}{$\begin{array}{c}\text { Generators in } \\
\text { Parallel mode }\end{array}$} & \begin{tabular}{c}
$|c|$ \\
NER \\
\cline { 2 - 3 }
\end{tabular} & $\begin{array}{c}\text { TH Neutral Current (A) } \\
\text { NERX } \\
\text { Reduction/ } \\
\text { Increase (\%) }\end{array}$ \\
\hline GTG-A & $7.114 \Omega)$ & 1.546 & $-78.27 \%$ \\
\hline
\end{tabular}

Table 9. Percentage reduction/increase of fault current between NER and selected NERX - parallel mode.

\begin{tabular}{|c|c|c|c|}
\hline \multirow{2}{*}{$\begin{array}{c}\text { Generators in } \\
\text { Parallel mode }\end{array}$} & $\begin{array}{c}|c| \\
\text { NER } \\
(31.75 \Omega)\end{array}$ & $\begin{array}{c}\text { Fault Current (A) } \\
(0.175 \mathrm{H} \text { or fundamental }=54.98\end{array}$ & $\begin{array}{c}\text { Reduction/ } \\
\text { Increase (\%) }\end{array}$ \\
\hline GTG-A & 175.7 & 100.3 & $-42.91 \%$ \\
\hline
\end{tabular}




\section{Conclusion}

NERX as neutral grounding can effectively reduce the TH current at the generator neutral. A slight increase in TH current is recorded during island mode due to a possible occurrence of series resonance with the system shunt capacitance. A drawback from using the chosen value of NERX is tolerable reduction in earth fault current at generator neutral during both operations. The findings in this paper would help to provide a solution toward NER overheating problem at UTP GDC plant. Further study should be conducted on resonance effect and transient overvoltage when using NERX to increase its effectiveness in mitigating TH current without compromising the primary function of limiting earth fault current.

The authors would like to thank MOHE in providing FRGS grant for the research and UTP for their support.

\section{References}

1. E. O'Neill-Carrillo, Power Systems Conference and Exposition, (2009).

2. Z. Ding, Y. Zhu, and C. Chen, 5th International Conference on Critical Infrastructure (CRIS), (2010).

3. N. Shah., Siemens Industry, Inc. (2013).

4. D. Chen, X. Chen, and M. Kang, International Conference on Electricity Distribution (CICED), 1318 (2014).

5. G. Angst and J. L. Oldenkamp, T. Am. Ins. Electrical Eng. Part III: Power Apparatus and Systems, 75, 1, (1956).

6. M. F. bin Abdullah, N. Hisham bin Hamid, Z. bin Baharudin, M. F. Irzaq bin Khamis, N. S. Rashidah binti Hashim, and S. bin Yusof, IPEC, 642 (2010).

7. Roger C. Dugan, Mark F. McGranaghan, Surya Santoso, H. Wayne Beaty, Electrical Power Systems Quality, McGraw-Hill, (2003).

8. L. J. Powell, IEEE T. Ind. Appl., IA-9, 672 (1973).

9. IEEE Guide for the Application of Neutral Grounding in Electrical Utility Systems. Part II - Grounding of Synchronous Generator Systems, IEEE Std, (1989).

10. M. F. B. Abdullah, Z. Baharudin, and N. H. B. Hamid, IEEE T. Energy Conver., 29, 519 (2014).

11. V. K. Mehta and R. Mehta, Principles of Power System: Including Generation, Transmission, Distribution, Switchgear and Protection : for B.E/B.Tech., AMIE and Other Engineering Examinations: S. Chand, (2005).

12. M. F. Abdullah, N. H. Hamid, Z. Baharudin, M. H. S. A. Razak, and I. B. Hisham, IPEC, 2012 Conference on Power \& Energy, 380 (2012).

13. IEEE Recommended Practice for Electric Power Distribution for Industrial Plants, IEEE Std 141-1993 (1994).

14. D. R. Adams, A. Feliachi, and W. L. Cooley, 12th WVU International Mining Electrotechnology Conference, 95 (1994).

15. M. F. Abdullah, N. H. Hamid, Z. Baharudin, and M. F. I. Khamis 4th International Conference Modeling, Simulation and Applied Optimization (ICMSAO), (2011). 